\title{
CALIDAD DE ATENCIÓN MÉDICA Y PRINCIPIOS ÉTICOS
}

\author{
Gabriel d’Empaire*
}

Resumen: La calidad en la atención se ha transformado en un requisito fundamental de todo servicio sanitario. A pesar del extraordinario desarrollo científico-tecnológico logrado en las últimas décadas, la complejidad de los servicios de salud ha hecho que se incrementen los errores médicos con consecuencias muy negativas en la morbilidad, mortalidad y costos de atención. Han surgido diferentes modelos de calidad de atención. Sin embargo, por lo general estos se implementan como procesos administrativos, gerenciales y alejados de la realidad del personal que los debe utilizar. La calidad de atención debe ser percibida no sólo como un problema médico gerencial, sino como un problema moral y, como tal, estar sustentado en una educación en valores que garantice la participación activa de todos los actores del sistema, siendo la excelencia, la solidaridad y el profesionalismo valores fundamentales.

Palabras clave: calidad de atención, bioética

\section{HEALTH CARE QUALITY AND ETHICAL PRINCIPLES}

Abstract: Health care quality has become a fundamental requirement for any health care service. Despite the extraordinary scientific-technological development achieved in the last decades, the complexity of health care services provokes the increase of medical errors with very negative consequences in morbidity, mortality and health care costs. Several health care quality models have been raised. Nevertheless, in general these are implemented as administrative, management processes far from the actual work of staff which must use them. Health care quality must not be perceived only as medical management problem, but as an ethical problem and, as such, it must be based on education on values which guarantee active participation of all stakeholders, being excellence, solidarity and professionalism fundamental values.

Key words: Health care quality, bioethics

\section{QUALIDADE DE ATENÇÃO MÉDICA E PRINCIPIOS ÉTICOS}

Resumo: A qualidade na atençáo transformou-se num requisito fundamental de todo serviço sanitário. Apesar do extraordinário desenvolvimento científico-tecnológico logrado nas últimas décadas, a complexidade dos serviços de saúde faz com que se incrementem os erros médicos com consequências muito negativas na morbilidade, mortalidade e custos de atenção. Têm surgido diferentes modelos de qualidade de atenção. Entretanto, em geral estes se implementam como processos administrativos, gerenciais, afastados da realidade do pessoal que deve utilizá-los. A qualidade de atenção deve ser percebida não somente como um problema médico gerencial, senão como um problema moral e, como tal, deve estar sustentada numa educação de valores que garanta a participação ativa de todos os atores do sistema, sendo a excelência, a solidariedade e o profissionalismo os seus valores fundamentais.

Palavras-chave: qualidade de atenção, bioética

\footnotetext{
Médico Cardiólogo Intensivista, Magíster en Bioética, Universidad Central de Venezuela. Hospital de Clínicas, Caracas, Venezuela
} Correspondencia: gabrieldempaire@yahoo.com 


\section{Introducción}

El tema de calidad de atención en los servicios de salud se ha transformado en uno de los aspectos fundamentales de la medicina actual. Los innumerables problemas surgidos durante las últimas décadas, derivados de la creciente complejidad de los sistemas de salud, aunados a las mayores exigencias por parte de los enfermos y sus familiares, así como los incrementos en los costos de atención, exigen soluciones efectivas que garanticen que los enfermos reciban los tratamientos adecuados, en el momento oportuno y dentro de un marco de equidad $\mathrm{y}$ respeto a sus valores y derechos.

La magnitud y el incremento de los problemas que enfrenta la medicina actual se equiparan en cierta forma a la dimensión y velocidad con la que se ha desarrollado la tecnología durante las últimas décadas. Desafortunadamente, la velocidad del cambio ha sido tal que a muchos, deslumbrados ante las oportunidades que brindan los recursos tecnológicos, les resulta difícil distinguir entre los beneficios y los problemas surgidos, y por tanto no logran captar la realidad de la situación. Nadie duda de los beneficios de los avances de la ciencia y tecnología; sin embargo, existen serias dudas sobre la efectividad de muchos de los tratamientos disponibles: muchos pacientes no reciben los que requieren en el momento en que los necesitan; se han incrementado en forma muy importante los errores en la atención de los enfermos; con frecuencia se violentan los derechos de los pacientes y han aumentado los costos de atención médica, con el consecuente crecimiento de los problemas de inequidad.

Ante esta realidad se han propuesto diferentes modelos para mejorar la calidad de atención médica, todos con el objetivo de resolver los problemas planteados en la medicina actual. Cada modelo tiene ventajas y desventajas, sin que hasta el momento exista uno solo que en forma consistente ofrezca un nivel de atención de alta calidad.

En los próximos párrafos presentaré, en primer lugar, algunos aspectos relacionados con los cambios que, a través de la historia, han determinado la necesidad de establecer estándares de calidad en la medicina, seguidos de una perspectiva clínica del problema. Luego, abordaré la relación entre calidad de atención y principios de la bioética como elemento fundamental para la adecuada implementación de cualquier modelo de calidad de atención médica. Si bien es indudable la responsabilidad que tienen los Estados en la calidad de atención como protección de los derechos humanos de los enfermos, no es mi intención entrar ahora en el análisis de este problema; por tanto, me limitaré a los aspectos de la calidad de atención relacionados con el personal de salud, los enfermos, sus familiares y la sociedad.

\section{De la excelencia médica a la calidad de atención de los sistemas de salud}

\section{Excelencia profesional}

Durante más de 24 siglos a los médicos se les ha exigido excelencia y profesionalismo basados en principios y valores morales. Tal solicitud ha sido claramente expresada a lo largo de la historia en diferentes juramentos y códigos deontológicos, a través de los cuales las sociedades han buscado garantizar una conducta médica apegada a las más estrictas exigencias morales.

El médico debe resolver en su quehacer diario problemas que comprometen los valores más sagrados del ser humano: la vida, la salud, la enfermedad, la felicidad y la capacidad de continuar con un proyecto de vida. De ahí que desde los inicios de la medicina occidental el médico fue considerado un "profesional", según la concepción histórica de la palabra. De acuerdo con Diego Gracia, "las profesiones son actividades muy particulares que obligan no sólo 'al bien hacer' sino a la 'perfección”" (1). Según esta concepción, las profesiones, a diferencia de los oficios, poseían un tipo de responsabilidad fuerte o responsabilidad moral, mientras que los oficios tenían responsabilidad jurídica. La responsabilidad moral protegía a estos profesionales de la responsabilidad jurídica, de forma que un profesional responsable era inmune. Esta condición profesional propiamente dicha, con las características antes descritas, estaba prácticamente reservada a tres actividades: el sacerdocio, la realeza y la medicina(1:52).

A esta condición profesional del médico se agregaba una connotación sagrada. Para Galeno: "Si el enfermo no admira al médico de algún modo como a un Dios no aceptará sus prescripciones" (1:81).

Esta connotación sagrada y suprajurídica en términos de la responsabilidad profesional exigía indudablemente perfección. Del médico siempre se ha esperado la perfección y la excelencia. Sin embargo, durante muchos siglos esta exigencia estuvo centrada en la figura del médico y en su quehacer profesional. El acto médico se llevaba a 
cabo dentro de un marco de relación íntima, privada, paternalista, según el cual el médico tenía la obligación de actuar y el paciente la de obedecer. A pesar de su elevada responsabilidad, contaba con escasos recursos y sus posibilidades de intervenir sobre las causas y mecanismos de la enfermedad eran muy limitadas. En este contexto, aplicaba sus conocimientos y técnicas en un acto solitario, siempre con la intención de aliviar al paciente, pero consciente de sus limitaciones. La restitución del orden natural establecía la línea de acción, a la vez que los conceptos de calidad de vida y de libertad para decidir no se solían incluir en la toma de decisiones.

\section{2. ¿Qué ha cambiado?}

$\mathrm{Al}$ menos dos grandes cambios se han suscitado en el tiempo: en primer lugar, la concepción clásica tradicional de la profesión perdió algunos de los aspectos que la caracterizaron, específicamente la inmunidad y los privilegios. Ya nadie puede ser inmune por su condición profesional ni gozar de una moralidad especial(2).

A su vez, en tan sólo pocas décadas la medicina cambió. Un extraordinario desarrollo científico tecnológico ha venido dotando al médico de innumerables recursos de diagnóstico y tratamiento. Hoy es posible tratar enfermedades que hasta hace poco tiempo no era posible hacerlo y en ocasiones ni siquiera aliviar. La atención médica se desarrolla en diferentes niveles, desde pequeños consultorios y centros ambulatorios hasta complejos centros hospitalarios. Estos últimos, en particular, han desarrollado complejos niveles de funcionamiento. Allí los enfermos son atendidos en ambientes dotados de novedosos recursos tecnológicos, por varios médicos (internos, residentes y diferentes especialistas). Además, participa personal de enfermería, técnicos, laboratoristas, farmacéuticos y personal administrativo, los cuales deben interactuar para la atención oportuna de cada paciente en particular, cada uno de ellos con problemas específicos que requieren un alto nivel de desempeño organizacional.

Dentro de este complejo sistema, por ejemplo, cada indicación médica debe cumplir diferentes pasos a través de un largo recorrido. El proceso se inicia con una decisión del médico respecto de un tratamiento particular. Con frecuencia, este primer paso encierra un alto nivel de complejidad y de responsabilidad, en la medida en que la selección del tratamiento debe hacerse, por lo general, dentro de un amplio espectro de posibilidades terapéuticas. Para esta selección el mé- dico debe contar con un elevado nivel de capacitación que le permita analizar, en forma crítica, una amplia y cambiante literatura -llena de resultados con frecuencia contradictorios, basados en complejos análisis estadísticos, a veces influidos por conflictos de interés-, de la cual debe extraer el tratamiento que, de acuerdo con las evidencias, resulta el mejor para ese enfermo(3). Una vez realizada la selección se inicia el recorrido que involucra personal de enfermería, farmacia, mensajeros hasta llegar finalmente al paciente, recorrido en el cual debe garantizarse el cumplimiento exacto de la indicación: dosis, dilución adecuada, conservación, vía y forma de administración, interacción con otras drogas y horario exacto de administración.

La complejidad de estos sistemas exige una nueva concepción que garantice el adecuado funcionamiento y control de cada uno de los procesos que se llevan a cabo en la atención de los enfermos. En otras palabras, el establecimiento de procedimientos que avalen una adecuada calidad de atención. En la medicina de hoy, la adecuada atención del enfermo va más allá de la excelencia individual del médico que lo atiende, la calidad de atención debe incorporar todos los elementos que forman parte del complejo sistema de atención médica. La excelencia del médico es un elemento indispensable pero no suficiente para logra una atención de calidad. La misma debe entenderse como parte de un concepto mucho más amplio, en el cual la excelencia es parte de una maquinaria que debe funcionar dentro de estándares muy estrictos, hoy conocidos como "estándares de calidad".

Si bien la excelencia y el profesionalismo han sido la tradición del médico durante muchos siglos, el concepto de calidad es un concepto mucho más reciente, sus orígenes datan del comienzo de los procesos industriales en las primeras décadas del siglo XX, cuando comenzaron a establecerse parámetros para la evaluación de los productos manufacturados. Posteriormente, economistas y sociólogos comenzaron a aplicar los conceptos de calidad en la vida humana, buscando índices que permitieran evaluar la calidad de vida de las personas y las sociedades. Y desde una época aún más reciente, los conceptos de calidad han comenzado a aplicarse a los sistemas de salud.

Si comparamos la Declaración Universal de los Derechos Humanos de 1948 con declaraciones más recientes, como la de Oviedo de 1997 y la Universal sobre Bioética y Derechos Humanos de la UNESCO, aprobada por unanimidad en 2005, veremos que en la primera se habla del derecho a la salud y a la aten- 
ción médica, mientras que en los otras dos se incluye el concepto de calidad de atención. Ya no es posible hablar solamente de un derecho a la salud o a la atención médica, es necesario que esa atención cumpla con estrictos criterios de calidad.

\section{3. ¿Qué es calidad de atención?}

En 1980 Donabedian describió la calidad de atención como: "Aquel tipo de cuidado en el cual se espera maximizar un determinado beneficio del paciente, luego de haber tomado en cuenta un balance de las ganancias y pérdidas implicadas en todas las partes del proceso de atención"(4).

En 1990 el Instituto de Medicina de Estados Unidos, parte de la Academia Nacional de Ciencias de ese país, propuso una definición de calidad que ha sido ampliamente aceptada: "Calidad de atención es el grado en el cual los servicios de salud para individuos y la población incrementan la posibilidad de obtener resultados deseados en salud y que sean consistentes con el conocimiento profesional médico"(5).

Existen diferentes aproximaciones al control de la calidad de atención (tabla 1). Algunos están basados en educación médica, guías de prácticas clínicas y medicina basada en evidencia. Otros utilizan modelos de autorregulación y certificación. Hay modelos basados en evaluación y feedback, mientras otros centran su atención en el paciente y el respeto a su autonomía y derechos. Finalmente, existen modelos basados en el diseño y control de procesos.
La mayoría de los modelos de calidad de atención están organizados como procesos administrativos, gerenciales, con lo cual, en muchas ocasiones, quedan desligados de la realidad clínica y especialmente alejados del personal que los debe utilizar, quienes, por lo general, desconocen las razones y la importancia de estos procesos.

\section{¿Qué está pasando?}

Los avances científico-tecnológicos de las últimas décadas han tenido un enorme impacto en mejorar la cantidad y calidad de vida de muchos pacientes, pero a la vez han generado numerosos problemas con consecuencias muy negativas, los cuales también debemos conocer y enfrentar. Una buena parte de estos problemas afectan de una u otra manera la calidad de atención sanitaria.

Uno de los aspectos más significativos radica en la enorme diferencia entre el conocimiento científico actual y la aplicación de esos conocimientos en la práctica clínica. El conocimiento ha crecido en forma realmente extraordinaria, pero en buena medida no se aplica en la práctica médica diaria. Como consecuencia, muchos pacientes no reciben los tratamientos que deben recibir y otras veces reciben tratamientos cuyo beneficio no está demostrado. A la vez, se han incrementado los errores médicos con graves consecuencias sobre la morbilidad, mortalidad y los costos de atención médica.

El acceso a la atención medica sigue siendo deficiente. En algunos países desarrollados millones de personas

Tabla 1(6)

\begin{tabular}{|l|l|}
\hline $\begin{array}{l}\text { Aproximaciones a la mejoría de la } \\
\text { calidad de atención }\end{array}$ & \\
\hline Aproximación & Asunciones \\
\hline $\begin{array}{l}\text { Medicina basada en la evidencia } \\
\text { Guías de prácticas clínicas } \\
\text { Ayuda para decisiones }\end{array}$ & La disponibilidad de la mejor evidencia, permite decisiones óptimas y un cuidado óptimo \\
\hline $\begin{array}{l}\text { Educación y desarrollo profesional } \\
\text { Autorregulación } \\
\text { Recertificación }\end{array}$ & $\begin{array}{l}\text { El aprendizaje basado en experiencia práctica y educación individual determina cambios } \\
\text { favorables en la actuación }\end{array}$ \\
\hline $\begin{array}{l}\text { Evaluación y responsabilidad } \\
\text { Feedback } \\
\text { Reportes públicos }\end{array}$ & $\begin{array}{l}\text { Provee feedback sobre los procedimientos entre grupos, reportes públicos de los datos, lo cual } \\
\text { motiva a cambios en las rutinas }\end{array}$ \\
\hline $\begin{array}{l}\text { Atención centrada en el paciente } \\
\text { Involucrar al enfermo } \\
\text { Compartir decisiones }\end{array}$ & Autonomía del enfermo y control sobre la enfermedad y los procesos \\
\hline $\begin{array}{l}\text { Calidad total } \\
\text { Mejoría continua de la calidad } \\
\text { Reestructuración de procesos }\end{array}$ & La mejoría de la calidad viene dada por la mejoría del proceso y no por cambios del individuo \\
\hline
\end{tabular}


no tienen acceso a la atención médica. El asunto es aún más grave en los países con escasos recursos, donde se presentan problemas de inequidad que resultan en graves complicaciones y muertes. Además, aun cuando exista disponibilidad, muchos tratamientos preventivos no están siendo adecuadamente utilizados, lo cual trae como consecuencia un incremento en enfermedades prevenibles y en complicaciones y muertes por estas enfermedades, además de la necesidad de utilizar más recursos para atenderlas. De la misma forma, muchos de los tratamientos de efectividad demostrada están siendo subutilizados.

De acuerdo con la Joint United Nations Program on HIVIAIDS (UNAIDS), 2008 Report(7), para finales de 2007 un estimado de 33,2 millones de personas vivían con VIH, de esas, 2,1 millones son niños y 2,1 millones murieron por SIDA. 6.800 personas se infectan diariamente con VIH debido a su poca capacidad de acceder a tratamientos de efectividad demostrada para prevenir la trasmisión de este virus. A pesar de todos los desarrollos científicos, la cobertura de la terapia retroviral permanece baja. Solamente el 31\% de las personas que lo requieren recibieron terapia antirretroviral en 2007. Ese mismo año, un estimado de 2,5 millones de personas fueron infectadas. Aun cuando desde hace varios años la trasmisión de madre a hijo se ha logrado prevenir, sólo la tercera parte de las mujeres embarazadas infectadas recibe drogas antirretrovirales para prevenir la trasmisión a sus hijos y aun menos reciben medicación para su propia salud.

La literatura reporta que alrededor de 27 millones de niños no reciben vacunas para prevenir diferentes enfermedades. A la vez, muchos pacientes no reciben tratamientos conocidos, basados en evidencias. Por sólo citar el caso de la enfermedad coronaria, primera causa de muerte en el hemisferio occidental, se sabe que alrededor de $30 \%$ de pacientes con cardiopatía isquémica no reciben Aspirina ${ }^{\circledR}, 50 \%$ de pacientes con enfermedad coronaria no reciben betabloqueantes. Solamente $37 \%$ recibe estatinas y menos del $40 \%$ mantiene un LDL menor a 100. Todos, tratamientos que han demostrado su efectividad en reducir los eventos coronarios y la mortalidad por este tipo de patología. La falla en la administración de tratamientos de efectividad demostrada - por ejemplo, trombolíticos, betabloqueantes, Aspirina ${ }^{\circledR}$, inhibidores de enzima convertidora de la angiotensina- en pacientes con enfermedad coronaria causa 18.000 muertes al año en Estados Unidos(8). Se ha reportado que 79\% de pacientes ancianos con infarto del miocardio no recibe betabloqueantes, con lo cual la mortalidad de estos pacientes fue $75 \%$ mayor en dos años que aquellos que sí recibieron esta droga(9). De la misma forma se ha determinado la subutilización de tratamientos para la hipertensión, la depresión y el cuidado prenatal. La subutilización de estos tratamientos para la prevención primaria y secundaria es causa de complicaciones en muchos pacientes que luego ameritan tratamientos más complejos y más costosos.

Otras veces, los pacientes son sometidos a tratamientos bajo circunstancias en las cuales el riesgo supera los beneficios esperados. Algunos pacientes son sometidos a tratamiento muy costosos simplemente porque la tecnología está disponible, sin que existan evidencias razonables de beneficio para los enfermos. Diferentes tratamientos con efectos beneficiosos demostrados compiten con tratamiento de efectividad no demostrada e, incluso, con evidencias de resultados negativos. Tratamientos con evidencias de efectividad para una determinada patología son utilizados en otras patologías donde su utilidad aún no ha sido demostrada. La literatura reporta que los antibióticos son en ciertas ocasiones sobreutilizados, con lo cual se incrementan las reacciones adversas, la resistencia bacteriana y los costos, sin que exista un beneficio para el enfermo (10). También ha sido demostrado que procedimientos como la coronariografía, las endarterectomías carotideas, las endoscopias gastrointestinales y los marcapasos, en algunas circunstancias, son sobreutilizados.

Al final de la vida, hoy contamos con diferentes alternativas terapéuticas que permiten prolongarla, pero otras veces estas mismas técnicas pueden prolongar inútilmente la vida de muchos enfermos irrecuperables, aumentado el dolor, el sufrimiento y los costos de atención de estos pacientes. En la actualidad resulta frecuente observar que enfermos con muy mal pronóstico -y por tanto con posibilidades de recuperación o mejoría prácticamente nulas, tales como pacientes en estado vegetativo permanente, cáncer, SIDA o cirrosis hepática en etapa terminal- son sometidos a métodos de soporte vital en unidades de cuidados intensivos. Existen serias dudas sobre si pacientes con enfermedades avanzadas con mal pronóstico deben someterse a métodos de soporte vital o a tratamientos orientados a prolongar la vida, una vez que se conoce que su expectativa de vida es muy corta o en quienes, 
en caso de sobrevivir, quedarían en condiciones de muy pobre calidad de vida. Debido a estas dudas, muchos pacientes son sobre tratados hasta llegar al denominado "encarnizamiento terapéutico"(11).

Como consecuencia, a pesar de los importantes avances científico-tecnológicos disponibles, muchos pacientes fallecen por errores médicos. El Instituto de Medicina América reportó en 2000 que entre 44.000 y 96.000 pacientes fallecían al año por estos errores(12). Entendiéndose por error médico aquellas complicaciones previsibles que pudieron ser evitadas. Entre ellas, interacción de drogas, dosis y vías de administración equivocadas $(13,14)$. Ha surgido así una nueva paradoja: si bien el desarrollo científico-tecnológico ha aumentado, permitiéndonos tratar enfermedades que hasta hace poco no tenían tratamiento, han aparecido nuevos problemas que han incidido negativamente en la mortalidad y la morbilidad, y han incrementado las desigualdades e inequidades.

A los problemas mencionados se suma otro cambio de gran significación, un nuevo modelo de relación más horizontal sustituyó al clásico modelo vertical paternalista que predominó en la tradición médica durante muchos siglos. Esta nueva concepción en las relaciones sanitarias -la cual puede ser considerada como uno de los avances más relevantes en términos de la evolución moral- ha traído importantes beneficios en términos de igualdad y respeto de los derechos de los enfermos. Sin embargo, un significativo porcentaje de pacientes no recibe información adecuada y necesaria para participar en la toma de decisiones sobre su salud, tratamientos y métodos diagnósticos propuestos. En otras ocasiones, la información que reciben no la comprenden. $Y$ en otras no son consultados o aun cuando se les consulte su voluntad no es respetada(15-18).

Por otra parte, los sistemas de atención médica enfrentan uno de los retos más importantes en la historia de la medicina: el incremento de los costos de atención(19), que ha sobrepasado la capacidad de los presupuestos de los Estados, aun en los países más ricos(20), y que reduce las oportunidades de muchos pacientes de recibir la atención médica que requieren. Cada vez contamos con más tecnologías y recursos que pueden ser utilizadas en menos pacientes. Aun cuando se acepte que es imposible lograr una absoluta igualdad para el goce de la mejor atención, cada miembro de la sociedad, independientemente de su condición socioeconómica, debe tener igual acceso a la atención médica. Para que un sistema de salud sea justo y equitativo necesita, en primer lugar, ser eficiente en términos de costobeneficio. En otra palabras, una gestión eficiente de recursos limitados.

Ante esta situación se han planteado nuevas interrogantes: ¿cómo conciliar el uso de tecnologías de alto costo con los derechos de los enfermos, en sistemas sanitarios con presupuestos limitados, a la vez que se garantiza una atención de calidad equitativa? Hoy se sabe que ningún sistema sanitario puede financiar todos los potenciales tratamientos para todos los pacientes que pudieran beneficiarse de éstos. ¿Cómo distribuir estos recursos en forma equitativa? ¿Cómo enfrentar los problemas de inequidad en países con muy bajos recursos? Se requiere establecer las necesidades reales de la población, así como sus prioridades, y establecer también limitaciones en la prestación de servicios. Por tanto, más allá de las decisiones gerenciales, tendremos que respondernos las siguientes preguntas éticas: ¿̨debe toda la tecnología ser utilizada en todos los enfermos? ¿Deben todos los pacientes recibir tratamientos orientados a prolongar la vida? ¿Están siendo utilizadas las nuevas tecnologías para salvar vidas de enfermos con una razonable posibilidad de sobrevivir en condiciones de calidad de vida aceptables o, por el contrario, se están usando para el tratamiento de enfermos con muy poco o ninguna chance de recuperación en términos de expectativa y calidad de vida(21)? Todos estos aspectos deben ser considerados al hablar de calidad de atención.

Hoy se acepta que debe existir limitación de prestaciones sanitarias. Es necesario definir un mínimo ético igual para todos. La pregunta es ¡cuáles criterios deben utilizarse para establecer estas limitaciones en forma equitativa? De acuerdo con lo establecido en el Pacto sobre los Derechos Económicos, Sociales y Culturales, debe lograrse el más alto estándar de salud. ¿Qué significa el máximo estándar? ¿Qué vamos a considerar justo para la aplicación de los tratamientos médicos? Ninguna de estas preguntas tiene respuestas adecuadas todavía. Para responderlas se requiere comenzar por un intenso programa de educación dirigido a todo el personal de salud, pero además que incluya a los pacientes, sus familiares y a todos los miembros de la sociedad. Se requiere además una amplia deliberación sobre los conceptos de salud, enfermedad, bienestar, límites de la tecnología, con el fin de establecer estrategias que permitan definir las necesidades de salud en los sistemas 
sanitarios, así como los criterios para el establecimiento del mínimo ético en cada sociedad. Discutir también los criterios de limitación de prestaciones (rationing), los límites de los derechos de los enfermos en relación con el uso de los recursos sanitarios y, sobre todo, debatir sobre los fines de la medicina(2:79).

\section{Relación entre calidad y principios de la bioética}

La calidad de atención debe enfocar al menos cuatro aspectos fundamentales:

- Una adecuada prevención y/o tratamiento, basado en evidencias, el cual debe ser aplicado oportunamente.

- Se debe evitar o reducir los posibles daños primarios o secundarios.

- Se debe respetar la dignidad y derechos de los enfermos.

- Se debe garantizar una gestión de recursos eficiente.

Para lograr estos objetivos se requiere:

\section{Calidad técnica: hacer lo correcto correctamente (22)}

Esta condición tiene dos dimensiones: hacer lo correcto, lo cual implica una alta capacidad de decisión, y en segundo lugar hacerlo correctamente, para lo cual se requiere una elevada calidad de realización -habilidades, juicio y oportunidad (hacerlo a tiempo).

La selección de un tratamiento requiere decisiones correctas. Cada tratamiento debe seleccionarse a partir de una variedad de alternativas terapéuticas; debe tener una probabilidad razonable de ser efectivo, de acuerdo con las evidencias existentes, no para una patología determinada sino individualizado para un paciente con condiciones particulares. Esto requiere una elevada calidad de decisión, hacer lo correcto. Una vez seleccionado el tratamiento, es necesario que el mismo se cumpla correctamente, en términos de dosis, difusión, vía de administración, horario. En otras palabras, hacerlo correctamente. Lo mismo pudiera decirse de un procedimiento terapéutico, como sería el caso de un procedimiento quirúrgico. Debe existir una oportunidad razonable de que, entre las diferentes alternativas, la decisión tomada sea la más adecuada para el paciente y luego la misma debe ejecutarse con habilidad, destreza y oportunidad. Nunca habrá certeza absoluta, pero debe exigirse una probabilidad razonable de eficacia, de acuerdo con las evidencias disponibles.

\section{Calidad de interacción entre el equipo de salud y los pacientes y sus familiares}

Esto significa calidad en la comunicación entre el médico, el paciente, el equipo de salud y los familiares, con el fin de mantener la confianza, la empatía, el interés, la honestidad, la solidaridad, la sensibilidad y el respeto a los valores y principios de los enfermos.

\section{Adecuado manejo de los costos de atención}

Establecer las políticas necesarias a fin de lograr una gestión de recursos eficiente.

Estos tres aspectos se sustentan claramente en los cuatro principios clásicos de la bioética -hacer el bien, no hacer daño, respetar la autonomía del enfermo y actuar con justicia-, así como con los diferentes principios expresados más recientemente en la Declaración Universal sobre Bioética y Derechos Humanos: respeto a la dignidad y derechos humanos, maximizar el beneficio y minimizar los riesgos, respetar la autonomía y responsabilidad individual, el consentimiento de las personas capacitadas y de aquellas sin capacidad de decidir, el respeto por la vulnerabilidad y la integridad de las personas, la privacidad, la confidencialidad, la equidad, la justicia, la igualdad, el respeto por la diversidad cultural y el pluralismo, la solidaridad, la cooperación y la responsabilidad social.

La implementación de cualquier proyecto de calidad de atención debe ser fundamentada en principios éticos, porque no es sólo un problema administrativo, organizacional o gerencial, sino moral. Requiere organización y gerencia, pero como medios para lograr el cumplimiento de una responsabilidad moral. Los pacientes acuden a los servicios de salud para que se les atienda, se les diagnostiquen sus problemas y en la medida de lo posible se les resuelvan. Resolver no siempre significa curar, significa también mejorar o aliviar cuando no se pueda curar, y ayudar y consolar cuando las condiciones anteriores no son posibles. Los sistemas sanitarios deben cumplir con estas obligaciones.

La calidad de atención requiere los siguientes aspectos: 
- Adecuada prevención y/o tratamiento basado en evidencias, aplicado en el momento adecuado.

- Eliminar o reducir los daños primarios y secundarios que se puedan prevenir.

- Respetar la autonomía y derechos de los enfermos.

- Establecer una relación costo-beneficio razonable, sustentable y justa.

Resulta indispensable que el personal de salud conozca con todo detalle los problemas relacionados con los errores médicos y sus causas, así como la responsabilidad profesional, moral y legal. Pero sobre todo es indispensable que participe activamente en el diseño y desarrollo de los procesos. Es necesaria la motivación, el liderazgo y la responsabilidad por parte de todos los integrantes del equipo de salud.

A su vez, la educación debe extenderse a los pacientes y sus familiares, quienes deben conocer los problemas con el fin de colaborar en su solución. Parte de esa información debe estar dirigida a resaltar su responsabilidad moral como actores del modelo de salud. Los pacientes y familiares deben conocer sus derechos y sus responsabilidades y también los límites de la medicina actual. El diseńo de procesos sin la plena participación de todos los implicados se transforma en rutina de trabajo que, si bien mejora algunos aspectos, tienen un elevado riesgo de fracaso.

\section{Conclusión}

No hay dudas de que uno de los grandes retos de la medicina actual es la implementación de modelos de calidad de atención que garanticen que los enfermos reciban los tratamientos adecuados, en el momento oportuno, minimizando las posibilidades de error, en un ambiente de respeto a su dignidad y derechos, y que se garantice la justicia y la equidad.

Sin embargo, más que un problema gerencial debe verse como un problema ético y, como tal, debe promoverse a través de la educación en valores y principios, siendo la excelencia, la solidaridad y la responsabilidad profesional los valores fundamentales.

Nuestra responsabilidad moral se incrementa en la misma proporción en que se incrementan nuestros conocimientos. Cuando una persona está enferma y se complica o muere debido a una enfermedad que no es prevenible o curable no hay responsabilidad moral; sí cuando es prevenible o existe un tratamiento efectivo para su mejoría o curación.

Como bien dice Gracia: "Las vías clásicas de control de la ética profesional han resultado ser muy poco eficaces y hoy es preciso plantear el tema de la responsabilidad ética de los profesionales desde perspectivas nuevas y con criterios distintos de los clásicos, como son los de la calidad total y la excelencia, que ya parecen estar dando importantes frutos en otros campos" (23).

\section{Referencias}

1. Gracia D. Fundamentos de bioética. 2a ed. Madrid: Triacastela; 2008: 62.

2. Gracia D. Como arqueros al blanco. Madrid: Triacastela; 2004: 276.

3. D’Empaire G, D’Empaire ME. La ética de la indicación médica y la medicina basada en la evidencia. Clínica Médica HCC 2003; VIII: 30-35.

4. Donabedian A. Exploration in quality assessment and monitoring. En: Harbor A. (ed.) The definition of quality and approaches to its assessment. Mich: Health administration Press; 1980

5. Lohr KN. (ed.) Medicare: A strategy for Quality Assurance. Washington DC: National Academy Press; 1990.

6. Grol R. Improving the Quality of Medical Care Building Bridges Among Professional Pride, Payer Profit, and Patient Satisfaction. JAMA 2001; 286: 2578-2585.

7. Towards universal access: scaling up priority HIVIAIDS interventions in the health sector. Progress report 2008. Geneva: World Health Organization; 2008.

8. Chassin MR, Galvin RW. The urgent need to improve heath care quality. JAMA 1998; 280: 1000-1005.

9. Soumerai SB, McLaughlin TJ, Spiegelman D, et al. Adverse outcomes in elderly survivors of acute myocardial infarction. JAMA 1997; 277: 115-121.

10. Kollef M, Sherdan G, Ward S, Fraser V. Inadequate Antimicrobial Treatment of Infections. A Risk Factor for Hospital Mortality among Critically Ill Patients. Chest 1999; 115; 462-474. 
Calidad de atención médica y principios éticos - Gabriel d’Empaire

11. Juan Pablo II. Evangelio de la vida. Caracas: Trípode; 1995: 76.

12. Kohn LT, Corrigan JM, Donaldson MS, (eds.) To Err is Human: Building a Safer Health System. Washington DC: National Academy Press; 2000.

13. Juurlink D, Mamdani M, Kopp A, et al. Drug-Drug Interactions Among Elderly Patients Hospitalized for Drug Toxicity. JAMA 2003; 289: 1652-1658.

14. Lee TH. A Broaden Concept of Medical Errors. N Engl J Med 2002; 347: 1965-1967.

15. Azoulay E, Sprung Ch. Family-physician interactions in the intensive care unit. Critical Care Med 2004; 32: 23232328.

16. The SUPPORT principal Investigators. A controlled trial to improve care for seriously ill hospitalized patients. JAMA 1995; 274: 1591-1598.

17. Devictor DJ, Tinh Nguyen D. How the decision is made in French pediatric intensive care units. Crit Care Med 2001; 29: $1356-1361$.

18. D’ Empaire G. Limitación de medidas terapéuticas en pacientes hospitalizados en una unidad de cuidados intensivos. Clinica Médica HCC 2001; VI: 143-148.

19. Anderson G, Chalkidou K. Spending on Medical Care. More is better? JAMA 2008; 299: 2444-2445.

20. Weintraub W. Cardiovascular Health Care Economics. New Jersey: Humana Press; 2003.

21. Gafo J. Los principios de justicia y solidaridad en bioética. En Gafo J. El derecho a la asistencia sanitaria y la distribución de recursos. Madrid: Universidad Pontificia de Comillas; 1999: 153.

22. Blumenthal D. Quality of Health Care. Part 1 Quality of Care-What is it? N Eng J Med 1996; 335: 891-894.

23. Gracia D. Ética y Vida. Profesión Medica, Investigacion y Justicia Sanitaria. Colombia: El Búho; 1998: 56.

Recibido: 23 de junio de 2010

Aceptado: 15 de julio de 2010 